\title{
A MORTALIDADE INFANTIL NO BRASIL: SÉRIE HISTÓRICA ENTRE 1994-2004 E ASSOCIAÇÃO COM INDICADORES SOCIO- ECONÔMICOS EM MUNICÍPIOS DE MÉDIO E GRANDE PORTE
}

\author{
INFANT MORTALITY IN BRAZIL: HISTORICAL SERIES FROM 1994 TO 2004 AND ASSOCIATION \\ WITH SOCIOECONOMIC INDICATORS IN MEDIUM AND LARGE MUNICIPALITIES
}

Tatiana Konrad Fischer1, Daniel Lima¹, Rosiléia Rosa1', Denise Osório¹, Antonio Fernando Boing²

${ }^{1}$ Alunos. Pós-graduação (Mestrado). ${ }^{2}$ Docente. Departamento de Saúde Pública. Centro de Ciências da Saúde - Universidade Federal de Santa Catarina (UFSc). Programa de Pós-graduação em Saúde Pública.

Correspondência: Antonio Fernando Boing (boing@ccs.ufsc.br) Universidade Federal de Santa Catarina. Centro de Ciências da Saúde. Departamento de Saúde Pública - Campus Universitário. 88040-900 - Florianópolis, SC, Brasil

Fischer TK, Lima D, Rosa R, Osório D, Boing AF. A mortalidade infantil no Brasil: série histórica entre 1994-2004 e associação com indicadores socioeconômicos em municípios de médio e grande porte. Medicina (Ribeirão Preto) 2007; 40 (4): 559-66, out./dez.

RESUMO: Modelo do estudo: trata-se de um estudo descritivo. Objetivo: descrever a série histórica da mortalidade infantil no Brasil segundo a idade do óbito e os principais grupos de causas no período de 1994 a 2004. Também foi testada a associação da mortalidade infantil com indicadores socioeconômicos nos 297 municípios brasileiros com população superior a 80 mil habitantes. Métodos: os municípios foram divididos em quartis segundo as taxas de mortalidade infantil e aplicaram-se testes ANOVA e Kruskall-Wallis para identificar possíveis diferenças entre os grupos. Também foram descritos os coeficientes de correlação de Spearman entre a variável dependente e as independentes. Os dados foram oriundos do Sistema de Informações sobre Mortalidade (SIM) e do Sistema de Informações sobre Nascidos Vivos (SINASC). Resultados: entre 1994 e 2004 houve declínio nas taxas de mortalidade infantil e alteração nos grupos de principais causas de óbitos infantis. Quando analisados apenas os municípios brasileiros de médio e grande porte, identificou-se que as taxas de mortalidade infantil apresentaram acentuada variação. Identificou-se, ainda, que todas as variáveis sócioeconômicas investigadas apresentaram correlação estatisticamente significante com a mortalidade infantil. Conclusão: políticas de atenção ao parto, à saúde materna, planejamento familiar e que agreguem ações de prevenção de doenças e promoção da saúde, devem ser dirigidas prioritariamente aos municípios que apresentem piores indicadores socioeconômicos.

Descritores: Mortalidade Infantil. Mortalidade Neonatal Precoce. Mortalidade Pós-neonatal. Mortalidade Infantil Tardia. Fatores Socioeconômicos.

\section{1- INTRODUÇÃO}

A taxa de mortalidade infantil (TMI) mede o risco de morte de crianças menores de um ano de idade e é considerado como um dos mais sensíveis indicadores de qualidade de vida de uma população ${ }^{1}$, sendo capaz de expressar tanto os níveis de saúde de uma sociedade quanto seu grau de desenvolvimento. ${ }^{2}$
Durante as últimas décadas houve um sensível declínio das TMI em diversas regiões do mundo, especialmente em países industrializados. Porém a magnitude dessa queda apresentou padrões distintos nos diferentes países e grupos populacionais do mundo. ${ }^{3,4}$ Estudos realizados no Brasil também identificaram que em período recente houve declínio na taxa de mortalidade infantil. Estima-se que ela declinou entre 
17\% e 18\% (2,2 milhões de óbitos) na década de 1990, especialmente em razão da expansão de programas de saúde em larga escala. ${ }^{5}$ No entanto, da mesma maneira que no resto do mundo, há desigualdades nos óbitos infantis quando comparadas as regiões e os estratos socioeconômicos do país. ${ }^{1,6}$

As regiões sul e sudeste do Brasil apresentam o melhor desempenho em relação à mortalidade infantil, sendo suas taxas similares aos níveis de alguns países desenvolvidos. Já a região nordeste expressa um padrão muito distante do desejável e possível, especialmente devido a fatores como a falta de saneamento básico, a carência de assistência em saúde e a desnutrição. $^{7}$

Além das diferenças regionais, as desigualdades entre os estratos sociais são fortes no Brasil. Em 2000, a TMI entre os $20 \%$ mais pobres do país equivalia a mais que o dobro da taxa identificada entre os $20 \%$ mais ricos. ${ }^{8}$

Considerando-se a complexidade e o dinamismo dos processos que envolvem a mortalidade infantil, é necessário o seu contínuo acompanhamento, de modo que se possa dispor de informações que permitam a análise da situação de saúde em nível local e a adoção das medidas de promoção de saúde adequadas ${ }^{9}$. Além disso, o estudo da maneira como as condições de vida e de infra-estrutura se associam com a TMI é importante para embasar os processos decisórios das políticas sociais e econômicas mais amplas que visem, em última instância, a redução da magnitude e das desigualdades em saúde. ${ }^{2}$

Neste sentido, o presente trabalho tem como objetivos descrever a mortalidade infantil segundo seus componentes e grupos de causas no Brasil no período de 1994-2004 e investigar sua associação com indicadores socioeconômicos em municípios de médio e grande porte do país.

\section{2- MATERIAL E MÉTODOS}

Foi analisada a taxa de mortalidade infantil no Brasil segundo a idade da criança no momento do óbito e os principais grupos de causas no período de 1994 a 2004. Os grupos etários investigados foram: 0 a 6 dias (mortalidade neonatal precoce), 7 a 27 dias (mortalidade neonatal tardia), 28 a 364 dias (pós-neonatal) e, englobando todos os grupos, 0 a 1 ano (mortalidade infantil). Já os grupos de causas de óbitos incluídos no estudo foram: afecções perinatais, anomalias congênitas, doenças respiratórias, doenças infecciosas e parasitárias e as demais causas.
Em seguida, para testar a associação entre indicadores socioeconômicos e a mortalidade infantil, empregaram-se como unidades de análise os 297 municípios brasileiros cujas populações foram iguais ou superiores a 80.000 habitantes no último recenseamento realizado no país (ano 2000). Como a TMI é bastante sensível à qualidade dos registros, recomenda-se a sua análise em nível municipal apenas nos municípios de médio e grande porte por estes possuírem maior confiabilidade e estabilidade nos seus dados..$^{10}$ Neste caso, foi calculada a média das taxas de mortalidade infantil do triênio 2002-2004 a fim de minimizar a possível influência de variações aleatórias dos dados.

As informações sobre nascidos vivos e de óbitos infantis são de domínio público e foram coletadas no Sistema de Informações sobre Nascidos Vivos (SINASC) e no Sistema de Informações sobre Mortalidade (SIM), respectivamente, durante os meses de setembro e outubro de 2006.

As variáveis socioeconômicas (variáveis exploratórias) foram obtidas através de dados secundários do Atlas de Desenvolvimento Humano no Brasil e do Sistema de Informações sobre Orçamento Público em Saúde (SIOPS). Foram coletados dados referentes à proporção de população acima de 25 anos com menos de 8 anos de estudo (educação); a proporção de domicílios particulares com banheiro e água encanada (saneamento básico); o PIB (Produto Interno Bruto) per capita (renda); o índice de Gini (concentração de renda); o gasto per capita em saúde e a proporção de indigentes.

O índice de Gini mede o padrão de distribuição de renda em uma população. Esse indicador apresenta uma escala que vai de 0 (zero) a 1 (um), sendo zero a situação de completa igualdade de distribuição de renda e 1 (um) a situação de extrema desigualdade. ${ }^{11}$

O PIB per capita é um indicador econômico que mede o valor total das riquezas produzidas em determinada região (bens e serviços) em relação ao tamanho de sua população. É calculado, portanto, através da razão do Produto Interno Bruto do município pela sua população total.

A indigência expressa a situação em que uma família não possui renda per capita suficiente para adquirir uma quantidade mínima de alimento para sua subsistência, definida como cesta básica de alimentos. Essa cesta é o que define a linha de indigência e é composta por um rol de produtos restritos e que são determinados com base num cálculo do valor calórico per capita diário já normatizado. A proporção de indigentes é calculada pela relação entre o total de famí- 
lias com renda inferior à linha de indigência pelo total de famílias na região em estudo, multiplicado por $100 .{ }^{11}$

O saneamento básico dos municípios foi avaliado através da proporção de domicílios com banheiro e água encanada. Quanto ao nível educacional, empregou-se a proporção de indivíduos com idade superior ou igual a 25 anos com menos de oito anos de estudo.

Os municípios foram agrupados em quartis segundo os valores da TMI no triênio 2002-2004, sendo o grupo 1 aquele que compreende os $25 \%$ dos municípios com menor mortalidade infantil e o grupo 4 o que corresponde ao um quarto de municípios com maior mortalidade infantil. Para testar a diferença entre as médias das variáveis sócioeconômicas em cada quartil foi utilizado o teste de ANOVA ou o seu correspondente não paramétrico, Kruskall-Wallis, para as variáveis com distribuição assimétrica. Também foram descritos os coeficientes de correlação de Spearman entre a variável dependente e as independentes, além das medidas de tendência central e de dispersão. $\mathrm{O}$ pacote estatístico utilizado foi o Stata. ${ }^{9}$

\section{3- RESULTADOS}

Entre 1994 e 2004 ocorreram no Brasil aproximadamente 757 mil óbitos de menores de um ano de idade. A série temporal delineada na Figura 1 apresenta um declínio nas taxas de mortalidade infantil durante os 11 anos investigados, ressaltando-se a inversão dos valores de mortalidade infantil na faixa etária de 1 a 11 meses a partir de 1995, comparativamente aos valores das taxas na faixa de 0 a 6 dias, mudança esta que vem se mantendo até o ano de 2004.

A Figura 2 demonstra que durante todo o período investigado as afecções perinatais corresponderam pela maior parte dos óbitos, chegando a quase $60 \%$ de todas as mortes infantis ocorridas no Brasil em 2004. Com menores percentuais apareceram as anomalias congênitas, as doenças infecciosas e parisitárias e as doenças respiratórias, respectivamente.

Quando analisados apenas os municípios brasileiros de médio e grande porte no triênio 2002-2004, identificou-se que as taxas de mortalidade infantil apresentaram acentuada variação entre os mesmos.

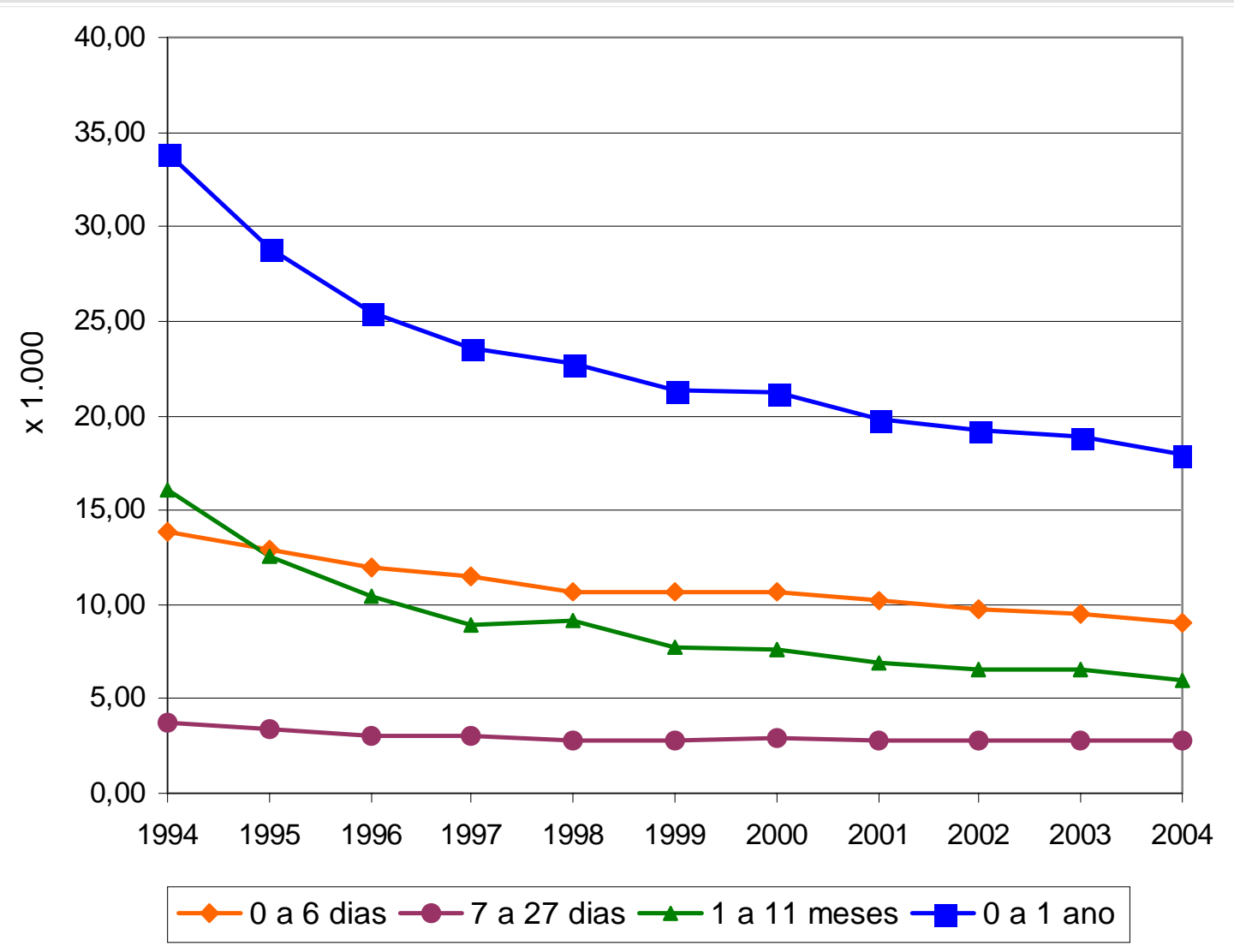

Figura 1: Série histórica das taxas de mortalidade infantil segundo seus componentes. Brasil, 1994-2004. 


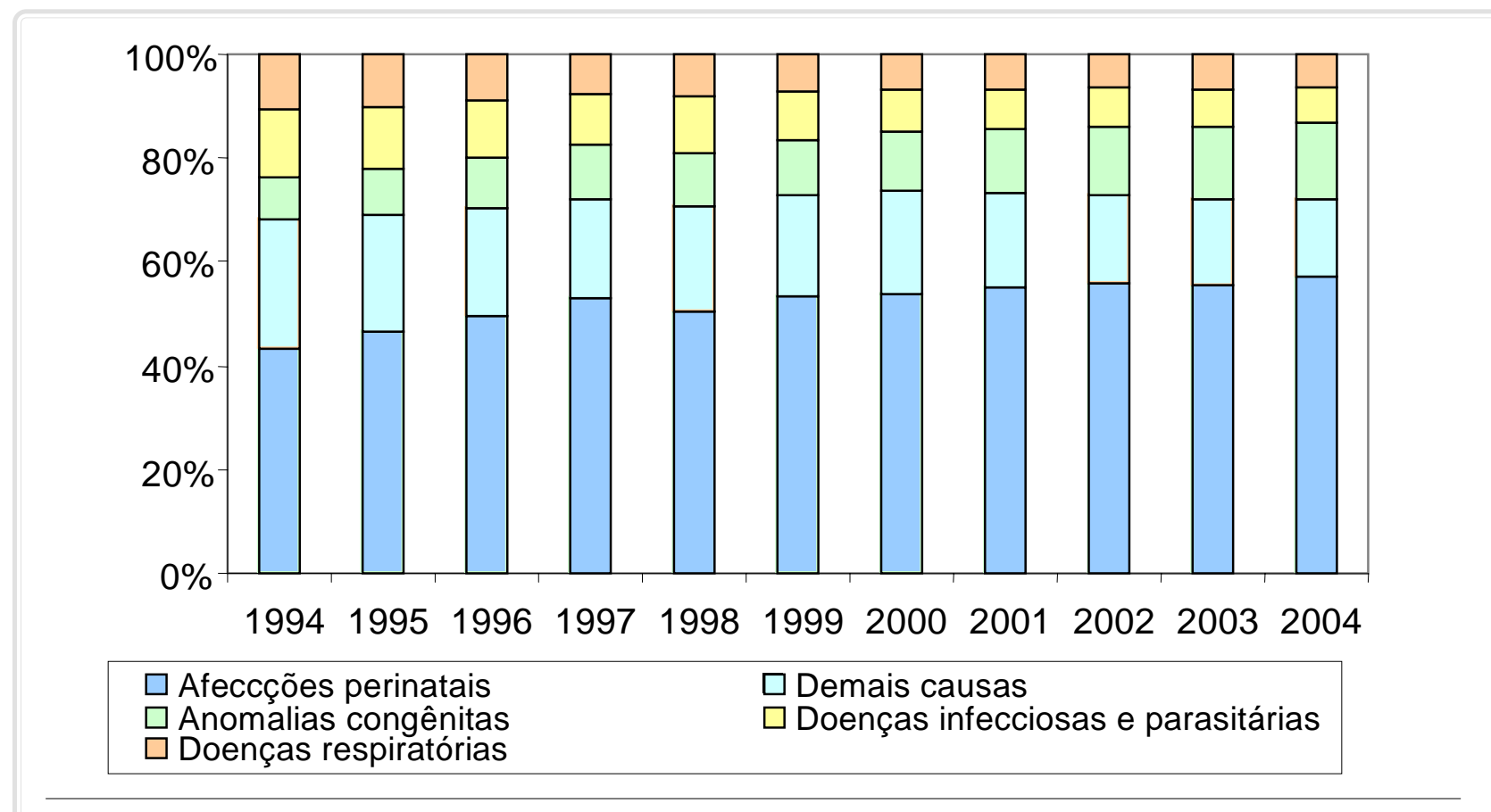

Figura 2: Proporção dos óbitos infantis segundo grupos de causas. Brasil, 1994 - 2004.

O valor mais baixo (5,78 óbitos por mil nascidos vivos) foi quase dez vezes inferior ao mais alto $(56,31$ óbitos por mil nascidos vivos), sendo a média identificada igual a 17,66 óbitos por mil nascidos vivos (Tabela I). Identificou-se, ainda, que todas as variáveis socioeconômicas investigadas apresentaram correlação estatisticamente significante com a TMI.

Na Tabela II está descrita a média dos indicadores socioeconômicos em cada quartil da mortalidade infantil. Comparando-se os grupos extremos (maior e menor TMI) identificou-se que os municípios no quartil com maior TMI apresentaram menor proporção de domicílios com banheiro e água encanada, maior proporção de pessoas com 25 anos ou mais com menos de oito anos de estudo, menor PIB per capita, menor gasto per capita em saúde, maior concentração de renda e maior proporção de indigentes $(\mathrm{p}<0,001)$.

\section{4- DISCUSSÃO}

Embora a qualidade dos registros oficiais no Brasil tenha melhorado nos últimos anos, é importante ressaltar as diferenças de valores encontrados entre os dados do Ministério da Saúde/DATASUS e do Instituto Brasileiro de Geografia e Estatística (IBGE). No ano de 2004, por exemplo, segundo o IBGE, a morta- lidade infantil chegou a 26,6 óbitos por mil nascidos vivos $^{8}$; já nos dados identificados neste estudo, com base nos registros do SIM, esta taxa foi de 17,90 óbitos por mil nascidos vivos.

As informações do IBGE são provenientes da transcrição das informações em mapas mensais pelos cartórios de Registro Civil que são enviados trimestralmente às superintendências estaduais do instituto e destas para o nível central. Os dados de óbitos do SIM têm como base as informações individualizadas das próprias declarações de óbitos.

Apesar da disponibilidade de dois sistemas de informações sobre óbitos em nível nacional, um dos problemas que ainda permeiam as análises de mortalidade no Brasil é o sub-registro de óbitos, que tem magnitude expressiva, sobretudo nas regiões menos desenvolvidas. A diferença entre o sistema IBGE e o SIM decorre também da recusa de alguns cartórios em enviar as declarações de óbitos ao órgão de saúde responsável por processar os dados e alimentar o SIM, uma vez que legalmente os cartórios são obrigados apenas a remeter os dados ao IBGE. ${ }^{12}$

Ao publicar pela primeira vez as Estatísticas de Mortalidade relativas ao ano de 1979, o Ministério da Saúde apontou que o Sistema de Informações de Mortalidade, de abrangência nacional, incluía apenas 
Tabela I: Estatística descritiva e coeficientes de correlação da taxa de mortalidade infantil e das variáveis sócioeconômicas. Municípios brasileiros com mais de 80 mil habitantes, 2000-2004

\begin{tabular}{|c|c|c|c|c|c|c|c|}
\hline Indicadores sociais & Média & Mediana & $\begin{array}{l}\text { Valor } \\
\text { mínimo }\end{array}$ & $\begin{array}{l}\text { Valor } \\
\text { máximo }\end{array}$ & 25 & 75 & $\begin{array}{c}\text { Coeficiente de } \\
\text { correlação }\end{array}$ \\
\hline Mortalidade Infantil & 17,66 & 16,91 & 5,78 & 56,31 & 13,92 & 20,65 & --- \\
\hline $\begin{array}{l}\% 25 \text { anos ou mais } \\
\text { com menos de oito } \\
\text { anos de estudo }\end{array}$ & 61,07 & 61,40 & 30,94 & 88,23 & 54,46 & 66,76 &, $324(* *)$ \\
\hline Índice de Gini & ,5621 & ,5600 & 43 & ,69 &, 52 & ,600 &, $387(* *)$ \\
\hline $\begin{array}{l}\% \text { de saneamento } \\
\text { básico }\end{array}$ & 85,58 & 93,22 & 12,32 & 99,19 & 81,02 & 97,12 &,$- 482(* *)$ \\
\hline PIB per capita & 304,36 & 290,48 & 71,06 & 834,00 & 209,95 & 382,69 &,$- 477(* *)$ \\
\hline $\begin{array}{l}\text { Gasto per capita em } \\
\text { saúde }\end{array}$ & 125,90 & 117,73 & 8,71 & 426,97 & 71,99 & 168,29 &,$- 218(* *)$ \\
\hline $\begin{array}{l}\text { Proporção de } \\
\text { indigentes }\end{array}$ & 11,25 & 8,11 & 1,16 & 51,11 & 4,92 & 14,05 &, $519(* *)$ \\
\hline
\end{tabular}

** Correlação é significante até o valor $p<0.01$

Tabela II: Distribuição das variáveis sócio-econômicas segundo o quartil de mortalidade infantil. Municípios brasileiros com mais de 80 mil habitantes, 2000-2004

\begin{tabular}{lccccc}
\hline Indicadores sociais & Quartil 1 & Quartil 2 & Quartil 3 & Quartil 4 & p-valor \\
\hline \% 25 anos ou mais com menos & & & & & \\
de oito anos de estudo & 56,76 & 58,17 & 62,96 & 66,34 & $<0,001$ \\
Índice de Gini &, 5462 &, 5507 &, 552 &, 5592 & $<0,001$ \\
\% de saneamento básico & 94,58 & 92,54 & 84,43 & 70,88 & $<0,001$ \\
PIB per capita & 395,37 & 344,50 & 262,33 & 216,44 & $<0,001$ \\
Gasto per capita em saúde & 149,84 & 136,21 & 109,08 & 109,09 & $<0,001$ \\
Proporção de indigentes & 6,14 & 8,07 & 11,37 & 19,37 & $<0,001$ \\
\hline
\end{tabular}

cerca de $70 \%$ do total de óbitos ocorridos naquele ano. A sub-enumeração de óbitos diz respeito, principalmente, à ocorrência de sepultamentos sem a exigência da certidão, nos denominados cemitérios clandestinos. $^{13}$

Em um estudo realizado em 1999, analisando a cobertura dos registros de óbitos para as unidades fe- derativas do Brasil no ano de 1990, observou-se que não há motivos para negligenciar as estatísticas de óbitos no Brasil. A afirmação de que elas são de má qualidade não serve como pretexto para ignorá-las, já que para muitas regiões do Brasil, elas possuem poder explicativo suficiente para construir indicadores de mortalidade com satisfatória confiança. ${ }^{14}$ 
O avanço na cobertura dos óbitos no Brasil é histórico e o maior salto nas coberturas possivelmente ocorreu durante o período de 1959 a 1980 . Durante o período de 1959 a 2000, o aumento da cobertura de óbitos no Brasil para ambos os sexos foi da ordem de $26 \%$, ou seja, um crescimento médio de $0,63 \%$ ao ano. Este aumento da cobertura de óbitos ocorreu em todas as regiões do Brasil, particularmente no norte e nordeste e mantendo-se a tendência é provável que todos os estados brasileiros superem a marca dos $80 \%$ de cobertura até o ano de 2010. ${ }^{15}$

As informações referentes aos nascimentos são obtidas através do SINASC (Sistema de Informação sobre Nascidos Vivos), cujo documento base é a declaração de nascimento, que tem emissão obrigatória no serviço de saúde onde ocorreu o parto. A cobertura do SINASC ainda apresenta deficiências embora a qualidade das informações venha melhorando desde a sua implantação. Estas deficiências têm menor magnitude do que as apresentadas pelo SIM. ${ }^{16}$

Recentemente, o Ministério da Saúde do Brasil tem estimulado o debate sobre a forma mais adequada de estimar a Mortalidade Infantil no país. ${ }^{17}$ Neste trabalho, no entanto, optou-se pela não aplicação de fatores de correção para as estimativas de óbitos e nascidos vivos a fim de expandir o acesso e a visibilidade dos dados oficiais do SIM e do SINASC e, assim, contribuir para a discussão acerca da qualificação destes registros.

Os dados encontrados refletem a notória modificação do padrão da distribuição dos óbitos infantis por grupos de causas. As afecções originadas no período perinatal passaram a predominar, possivelmente em decorrência da redução mais acentuada dos óbitos por doenças infecciosas e parasitárias e por doenças do aparelho respiratório. Tais fatos podem estar refletindo a influência da adoção de algumas medidas de atenção à saúde, como por exemplo programas de controle das infecções respiratórias agudas (IRA), a terapia de reidratação oral (TRO) e o incentivo à amamentação, conforme já assinalados em estudos anteriores. . $^{6,18,19,20}$

A queda da mortalidade infantil no Brasil também está associada a uma série de melhorias nas condições de vida e na atenção à saúde da criança em relação a questões como segurança alimentar e nutricional, saneamento básico e vacinação. No entanto, esses progressos não beneficiam a população de maneira uniforme, havendo desigualdades regionais. ${ }^{8}$ No presente estudo, os valores de TMI variaram de 5,78 (valor mínimo) a 56,31 (valor máximo) nos municípios pesquisados, o que reflete estas diferenças de maneira significativa. Para promover melhorias e eqüidade destaca-se a necessidade de priorização das intervenções nas regiões norte e nordeste, em particular nas áreas rurais; assegurar a sustentabilidade do progresso já alcançado nas áreas de imunização, terapia de reidratação oral e planejamento familiar; investir num melhor atendimento ao parto e na atenção pré-natal, assim como aumento da cobertura de pré-natal; investir no manejo de casos integrado de doenças infantis, planejamento familiar, promoção do aleitamento materno e vacina anti-Hib. ${ }^{19}$

Além da redução da mortalidade infantil que vem sendo observada, é evidente a associação das variáveis socioeconômicas utilizadas no presente estudo com as TMI, uma vez que todas as variáveis exploratórias deste estudo apresentaram diferentes médias entre os quartis, com significância estatística. Estudos ecológicos anteriormente realizados indicam estas relações.

Em Recife (PE) em 2003 foi detectada uma relação inversa entre a condição de vida e a magnitude da mortalidade infantil, revelando desigualdades ocultadas nos indicadores médios de saúde. Neste estudo, as variáveis socioeconômicas analisadas foram abastecimento de água, instalação sanitária, coleta de lixo, analfabetismo, anos de estudo, renda e densidade intradormitório. ${ }^{21}$ Em Salvador (BA), em um estudo ecológico de base populacional que descreveu a evolução da mortalidade infantil de 1991 a 1997 e analisou a relação entre a distribuição espacial da mesma com fatores socioeconômicos de renda, educação, saneamento e moradia, observou-se que apesar da redução dos níveis da mortalidade infantil, são mantidas as desigualdades sociais em saúde, sendo a baixa educação a variável de maior correlação com as piores taxas de mortalidade infantil. ${ }^{9}$

No presente estudo, também se observou que quanto maior a proporção de população acima de 25 anos com menos de 8 anos de estudo, maior a TMI no grupo. $\mathrm{O}$ grau de instrução das mulheres é de grande impacto sobre a mortalidade infantil, ressaltando que quanto maior o número de anos de estudo da mãe, mais chance ela tem de evitar a morte de um filho nos primeiro cinco anos. ${ }^{8}$

Outras relações entre a mortalidade infantil e indicadores socioeconômicos têm sido evidenciadas no Brasil, entre elas associação persistente entre os fatores socioeconômicos e os resultados adversos da gravi- 
dez (baixo peso ao nascer e óbito perinatal). ${ }^{22} \mathrm{Em}$ um estudo de base populacional realizado no município de Ribeirão Preto (SP), os resultados demonstraram que as áreas pobres da cidade apresentam TMI mais elevadas quando comparadas com áreas mais privilegiadas. ${ }^{23}$ Corroborando com estas afirmações, estudos evidenciaram a importância dos fatores ligados aos processos sociais e condições de vida, tais como educação, renda, saneamento básico e acesso a bens e serviços na determinação da mortalidade infantil. ${ }^{2,3}$ Fator também apontado pela literatura é o aumento das equipes de Programa de Saúde da Família (PSF) no Brasil, que contribuiu para a queda das TM..$^{\mathrm{I}, 24}$

O estudo da mortalidade infantil considerando sua distribuição no espaço geográfico contribui para demonstrar a manutenção das desigualdades sociais em saúde. Cabe salientar que o objetivo deste trabalho não foi o de determinar riscos à mortalidade infantil em nível individual, mas sim, descrever em relação ao contexto social em municípios agregados. Desta maneira, os resultados encontrados revelam que a TMI associa-se às condições de vida nos locais estudados, mostrando a necessidade de políticas econômicas e sociais abrangentes que objetivem a redução destas TMI no Brasil, principalmente considerando-se as disparidades regionais vigentes.
Não foi objetivo do presente estudo identificar as desigualdades intramunicipais, mas é útil para a formulação e implantação das políticas de saúde. Políticas de atenção ao parto, à saúde materna, ao planejamento familiar e que agreguem ações de prevenção de doenças e promoção da saúde, devem, respeitando o princípio da eqüidade do Sistema Único de Saúde, ser dirigidas prioritariamente aos municípios que apresentem piores indicadores socioeconômicos.

\section{5- CONCLUSÃO}

Identificou-se que entre 1994 e 2004 a mortalidade infantil no Brasil foi declinante. Dentre os principais grupos de causa houve predominância das afecções originadas no período perinatal e das anomalias congênitas em 2004, havendo declínio proporcional dos óbitos por doenças infecciosas e parasitárias e por doenças respiratórias.

No nível ecológico importante associação entre mortalidade infantil e indicadores socioeconômico foram identificados. Municípios com maior concentração de renda, maior proporção de indigentes, pior indicador de escolaridade e de saneamento básico e menor PIB per capita e de investimento em saúde apresentam maior mortalidade infantil.

Fischer TK, Lima D, Rosa R, Osório D, Boing AF. Infant mortality in Brazil: historical series from 1994 to 2004 and association with socioeconomic indicators in medium and large municipalities. Medicina (Ribeirão Preto) 2007 ;40 (4): 559-66, oct./dec.

ABSTRACT: Study design: descriptive study. Objective: to describe the historical series of infant mortality in Brazil according to age of death and main groups of causes, from 1994 to 2004. Association of socioeconomic indicators and infant mortality in 297 Brazilian municipalities with population equal or above 80.000 was also tested. Methods: the municipalities were divided into quartiles and the ANOVA and Kruskall-Wallis tests were applied to identify possible differences between the groups. Spearman's coefficient of correlation between the dependent and independent variables was also described. Data was obtained from the Mortality Information System (SIM) and the Live Birth Information System (SINASC), from the Brazilian Ministry of Health/Datasus. Between 1994 and 2004, there was a decline in infant mortality rates and changes in main causes of death among infants. Results: when observed only medium and large Brazilian municipalities, a high variation of infant mortality rates was detected. The study also revealed that all socioeconomic variables investigated presented a statistically significant correlation with infant mortality rates. Conclusion: specific policies on birth-care, maternal health, family planning and other actions to prevent illness and promote health should be directed with a higher priority to municipalities that present the worst socioeconomic indicators.

Keywords: Infant Mortality. Early Neonatal Mortality. Postneonatal Mortality. Late Infant Mortality. Socioeconomic Factors. 


\section{REFERÊNCIAS}

1 - Vermelho LL, Leal AJC, Kale PL. Indicadores de Saúde. In: Medronho R, editor. Epidemiologia. São Paulo: Atheneu; 2006. p.33-56.

2 - Andrade SM, Soares DA, Matsuo T, Souza RKT, Mathias TAF, Iwakura MLH et al. Condições de vida e mortalidade infantil no Estado do Paraná, Brasil, 1997/2001. Cad Saúde Pública 2006; 22(1):181-9.

3 - Rutstein SO. Factors associated with trends in infant and child mortality in developing countries during the 1990's. Bull World Health Organ 2000;78(10):1256-70.

4 - Wegman ME. Infant mortality in the 20th century, dramatic but uneven progress. J. Nutr 2001;131(2):401S-8S.

5 - Lopez AD. Reducing child mortality. Bull World Health Organ 2000;78(10):1173.

6 - Menezes AMB, Victora CG, Barros FC, Albernaz E, Menezes FS, Jannke HA et al. Mortalidade infantil em duas coortes de base populacional no Sul do Brasil: tendências e diferenciais. Cad Saúde Pública 1996; 12(Supl.1):S79-S86.

7 - Szwarcwald CL, Castilho EA. Estimativas da mortalidade infantil no Brasil, década de 80: proposta de procedimento metodológico. Rev Saúde Pública 1995;29(6): 451-62.

8 - Fundo das Nações Unidas para a Infância. Situação da infância brasileira, 2006. Brasília: UNICEF; 2005.

9 - Costa MCN, Azi PA, Paim JS, Silva LMV. Mortalidade infantil e condições de vida: a reprodução das desigualdades sociais em saúde na década de 90 . Cad Saúde Pública 2001;17(3):555-67.

10 - BRASIL. Ministério da Saúde. Portaria 21 de 5 de janeiro de 2005: Indicadores do Pacto da Atenção Básica. Brasília; 2005.

11 - Januzzi PM. Indicadores sociais no Brasil. São Paulo: Alínea; 2003.

12 - Mello-Jorge MHP, Gotlieb SLD. As condições de saúde no Brasil: retrospecto de 1979 a 1995. Rio de Janeiro: FIOCRUZ; 2000.

13 - Szwarcwald CL, Leal MC, Castilho EA, Andrade CLT. Mortalidade Infantil no Brasil: Belíndia ou Bulgária? Cad Saúde Pública 1997;13(3):503-16.
14 - Paes NA, Albuquerque MEE. Avaliação da qualidade dos dados populacionais e cobertura dos registros de óbitos para as regiões brasileiras. Rev Saúde Pública 1999; 33 (1):33-43.

15 - Paes NA. Avaliação da cobertura dos registros de óbitos dos estados brasileiros em 2000. Rev Saúde Pública 2005;39(6):882-90.

16 - Schramm JMA, Szwarcwald CL. Sistema hospitalar como fonte de informações para estimar a mortalidade neonatal e a natimortalidade. Rev Saúde Pública 2000;34(3):272-9.

17 - Szwarcwald CL, Leal MC, Andrade CLT, Souza Jr PRB. Estimação da mortalidade Infantil no Brasil: o que dizem as informações sobre óbitos e nascimentos do Ministério da Saúde? Cad Saúde Pública 2002;18(6):1725-36.

18 - Paim JS, Costa MC. Decline and unevenness of infant mortality in Salvador, Brazil, 1980-1988. Bull Pan Am Health Organ 1993;27(1):1-14.

19 - Victora CG. Intervenções para reduzir a mortalidade infantil pré-escolar e materna no Brasil. Rev Bras Epidemiol $2001 ; 4(1): 3-69$.

20 - Costa MCN, Mota ELA, Paim JS, Silva LMV, Teixeira MG, Mendes CMC. Mortalidade Infantil no Brasil em períodos recentes de crise econômica. Rev Saúde Pública 2003;37(6):669-706.

21 - Guimarães MJB, Marques NM, Melo Filho DA, Szwarcwald CL. Condições de vida e mortalidade infantil: diferenciais intra-urbanos no Recife, Pernambuco, Brasil. Cad Saúde Pública 2003;19(5):1413-24.

22 - Andrade CLT, Szwarcwald CL, Gama SGN, Leal M.C. Desigualdades sócio-econômicas do baixo peso ao nascer e da mortalidade perinatal no município do Rio de Janeiro, 2001. Cad Saúde Pública 2004;20(Supl. 1):S44-S51.

23 - Goldani MZ, Barbieri MA, Bettiol H, Barbieri MR, Tomkins A Infant mortality rates according to socioeonomic status in a Brazilian city. Rev Saúde Pública 2001;35(3):256-61.

24 - Macinko J, Guanais FC, Souza MFM. Evaluation of the impact of the Family Health Program on infant mortality in Brazil,19902002. J Epidemiol Comunnity Health 2006;60(1):13-9.

Recebido em 12/04/2007

Aprovado em 31/07/2007 